\title{
Curasept ADS the first 'non-staining' chlorhexidine supported by clinical evidence
}

Chlorhexidine is still considered to be the most effective oral antiseptic and is defined as the gold standard in this field. The formation of dental pigmentations is certainly the best known adverse side-effect of chlorhexidine. It might represent a serious limitation and reduce patient compliance with the prescribed therapy. However, in periodontology, compliance with therapy prescribed is fundamental for the clinical success in both the active and maintenance phases of treatment.

At the beginning of the 2000s in Italy, a patented system called Anti Discoloration System (ADS) was developed. This is contained in the Curasept ADS line of products.

The ADS system is able to interfere with the two main reactions that are responsible for stain formation, that is, the Maillard reaction and the protein denaturation process. Thanks to the absence of alcohol and SLS, the Curasept ADS line reduces pain, mucosal dehydration and irritations during rinsing. It helps in preventing the risk of developing side effects that are related to the prolonged use of alcohol-based mouthwashes, therefore, increasing compliance.

The efficacy of Curasept ADS against gum inflammation has now been confirmed by numerous in-vitro and in-vivo studies. In-vivo studies clearly demonstrate that the control of gingival inflammation is ensured by the constant use of Curasept ADS Mouthwash 0.2\% (for example Cortellini 2008, cross-over, 48 patients; Solis 2011, cross-over, 15 patients; Graziani 2015, controlled, 70 patients), even if all oral hygiene manoeuvres are suspended (Cortellini 2008; Solis 2011).

During the last Euro Perio 9 Congress, which took place in Amsterdam in June 2018, more evidence was presented in a tripleblind, parallel-arm, randomised controlled trial (Trombelli L, Farina $\mathrm{R}$ et al. 2018, 35 patients undergoing oral surgery consisting of the elevation of a full-thickness flap, evaluated for a 21-day period) that showed that $\mathrm{CHX}+\mathrm{ADS}$ leads to an excellent surgical wound healing quality and $\mathrm{CHX}+\mathrm{ADS}$ demonstrates powerful anti-plaque activity, comparable to that of conventional CHX $0.2 \%$ without ADS.

At the Congress a systematic review and meta-analysis, the highest level of scientific evidence, was also presented. The title was The efficacy of chlorhexidine mouthwash, with and without an Anti Discoloration System(ADS), on the parameters plaque, gingivitis and staining control. A systematic review and meta-analysis. The authors were B. W. M. Van Swaaij, G. A. Van Der Weijden, E. W. P. Bakker and D. E. Slot.

The aim was to determine whether combining chlorhexidine with ADS is efficacious in reducing tooth discolouration, whilst maintaining the plaque- and gingivitis-reducing effects of chlorhexidine.

Eighty-seven publications were analysed and 11 articles selected for review - six with protocols in which mechanical hygiene was discontinued, five in which mechanical hygiene was not discontinued. It was possible to obtain 14 direct comparisons, nine of which were included in the final meta-analysis.

The conclusion stated: 'Adding ADS to a chlorhexidine mouthwash significantly reduces tooth discolouration and does not affect the action of chlorhexidine in controlling gingival inflammation and plaque indices. The recommendation resulting from this review is that the chlorhexidine and ADS combination should be taken into consideration, given its efficacy in reducing plaque and gingival inflammation associated with anti-discolouration activity.'

Some studies suggested that the addition of the ADS system based on sodium metabisulphite and ascorbicacid, while reducing tooth staining, could also weaken the efficacy of chlorhexidine and limit its action (Li \& Lang, 2013; Guggenheim \& Meier, 2011).

However, it seems peculiar that other renowned authors have highlighted exactly the opposite, namely that chlorhexidine was fully functional, while the ADS system was totally or almost totally ineffective (Addy, 2005; Bevilacqua, 2016).

It should be emphasised that the components of the ADS system are not chemically able to interact with chlorhexidine in solution, therefore, they cannot weaken its efficacy.

Instead, when analysing the efficacy of an antiseptic mouthwash, as regards conflicting results of the studies conducted on Curasept ADS, it is possible to speculate that the choice of the protocol, the study population and the statistical analysis may affect result outcomes.

Therefore, we probably should evaluate more positively studies that investigate real-life clinical use of the test product in a statistically predetermined number of subjects rather than healthy volunteers who are not representative of real operating conditions.

Curasept ADS is a professional range of anti-bacterial mouthwashes, which contains a patented, clinically tested Anti Discolouration System (ADS) which, unlike other chlorhexidine mouthwashes, drastically reduces the appearance of brown-yellow spots which can form on the teeth due to chlorhexidine.

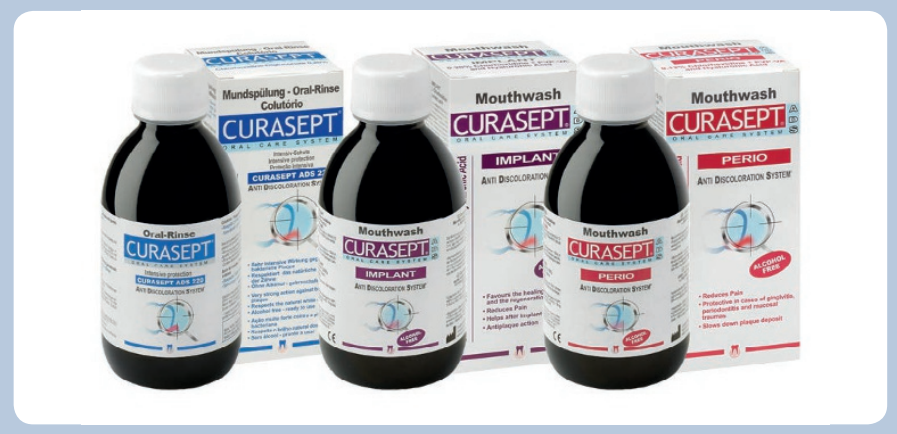

Take advantage of Curasept to increase your practice income - this is not just a product for helping your patients, this is a product for growing your practice. The Curasept ADS system comes with FREE patient oral care 'prescription' cards which give you the opportunity to easily encourage purchase by your patients, literature for the waiting room and training to optimise practice sales.

Available through your usual dental retailer or by contacting J\&S Davis directly on 01438747344. 